\title{
Boron And Carbon Cosmic rays in the Upper Stratosphere (BACCUS)
}

K. C. Kim* ${ }^{* 11}$, Y. Amare' ${ }^{1}$, T. Anderson ${ }^{2}$, D. Angelaszek ${ }^{1,3}$, N. Anthony ${ }^{3}$, K. Cheryian ${ }^{3}$, G.H. Choi $^{4}$, M. Copley ${ }^{1}$, S. Coutu ${ }^{2}$, L. Derome ${ }^{5}$, L. Eraud ${ }^{5}$, L. Hagenau ${ }^{3}$, J.H. Han ${ }^{1}$, H.G. Huh ${ }^{1}$, S. Im ${ }^{2}$, J.A. Jeon ${ }^{4}$, S. Jeong ${ }^{4}$, M.H. Kim ${ }^{1}$, H.Y. Lee ${ }^{4}$, J. Lee ${ }^{4}$, M.H. Lee ${ }^{1}$, J. Liang 3 , J.T. Link $^{6}$, L. Lu' ${ }^{3}$ L. Lutz ${ }^{1}$, T. Mernik ${ }^{1}$, J.W. Mitchell ${ }^{6}$, S.I. Mognet ${ }^{2}$, S.Morton ${ }^{3}$, M. Nester ${ }^{3}$, S. Nutter $^{7}$, O. Ofoha ${ }^{1}$, I.H. Park ${ }^{4}$, N. Picot-Clémente ${ }^{1}$, R. Quinn ${ }^{3}$, E.S. Seo ${ }^{1,3}$, J.R. Smith ${ }^{1}$, P. Walpole $^{1}$, R.P. Weinmann ${ }^{1}$, J.Wu ${ }^{1}$, Y.S. Yoon ${ }^{1}$

${ }^{1}$ Inst. for Phys. Sci. and Tech., University of Maryland, College Park, MD 20742, USA

${ }^{2}$ Dept. of Physics, Penn State University, University Park, PA 16802, USA

${ }^{3}$ Dept. of Physics, University of Maryland, College Park, MD 20742, USA

${ }^{4}$ Dept. of Physics, Sungkyunkwan University, Suwon 440-746, Republic of Korea

${ }^{5}$ Laboratoire de Physique Subatomique et de Cosmologie, UJF - CNRS/IN2P3 - INP, 53 rue des Martyrs, 38026 Grenoble Cedex, France

${ }^{6}$ Astroparticle Physics Lab., NASA Goddard Space Flight Center, Greenbelt, MD 20771, USA

${ }^{7}$ Dept. of Physics and Geology, Northern Kentucky Univ., Highland Heights, KY 41099, USA

The balloon-borne BACCUS experiment measures directly the elemental spectra of cosmic-ray nuclei from protons to Fe over the energy range $\sim 10^{12}$ to $10^{15} \mathrm{eV}$. It focuses on the energy dependence of secondary to primary ratios (e.g. B/C) to investigate cosmic-ray propagation history. BACCUS consists of redundant and complementary particle detectors including the Timing Charge Detector (TCD), Transition Radiation Detector (TRD), Cherenkov Detector (CD), Silicon Charge Detector (SCD), and Calorimeter (CAL). The TCD measures the light yield produced by the particle in plastic scintillator. The TRD provides energy measurements of incident $3 \leq \mathrm{Z} \leq 26$ nuclei in the $10^{2}-10^{5}$ Lorentz factor range. The CD responds only to particles with velocity exceeding the velocity of light in the plastic. It allows BACCUS to reject the abundant low energy cosmic rays present in the polar region. The CAL is used to determine the particle's energy for all nuclei for $1 \leq Z \leq 26$. With the SCD based on pixellation, in addition to the TCD based on timing, and the $\mathrm{CD}$, the BACCUS instrument implements virtually all possible techniques to minimize the effect of backscatter on charge measurements in the presence of a large particle shower in the CAL. The 30 day flight was carried out successfully over Antarctica in 2016 from Nov. 28 to Dec. 28. The integration test, and performance of instruments are presented.

35th International Cosmic Ray Conference - ICRC2017

10-20 July, 2017

Bexco, Busan, Korea

*Speaker
${ }^{\dagger}$ kckim@umd.edu




\section{Introduction}

The Boron And Carbon Cosmic rays in the Upper Stratosphere (BACCUS) is a balloonborne experiment to directly measure the elemental spectra from proton to iron nuclei with energies $\sim 10^{12} \sim 10^{15} \mathrm{eV}$. It is a successor of the CREAM experiment [1] already launched six times from McMurdo polar station, Antarctica, since December 2004. The measurements of the high energy cosmic nuclei will continue to be carried out by the BACCUS instrument, similarly, in a series of annual balloon flights. BACCUS, however, mainly focuses on the energy dependence of secondary to primary ratios (e.g. B/C) to investigate cosmic-ray propagation history as well as acceleration mechanisms and their sources.

The instrument comprises of TCD, TRD, CD, SCD, Hodoscope (S3) and CAL [2]. Fig. 1 shows the BACCUS instrument. The integration tests and performance of instruments are presented.

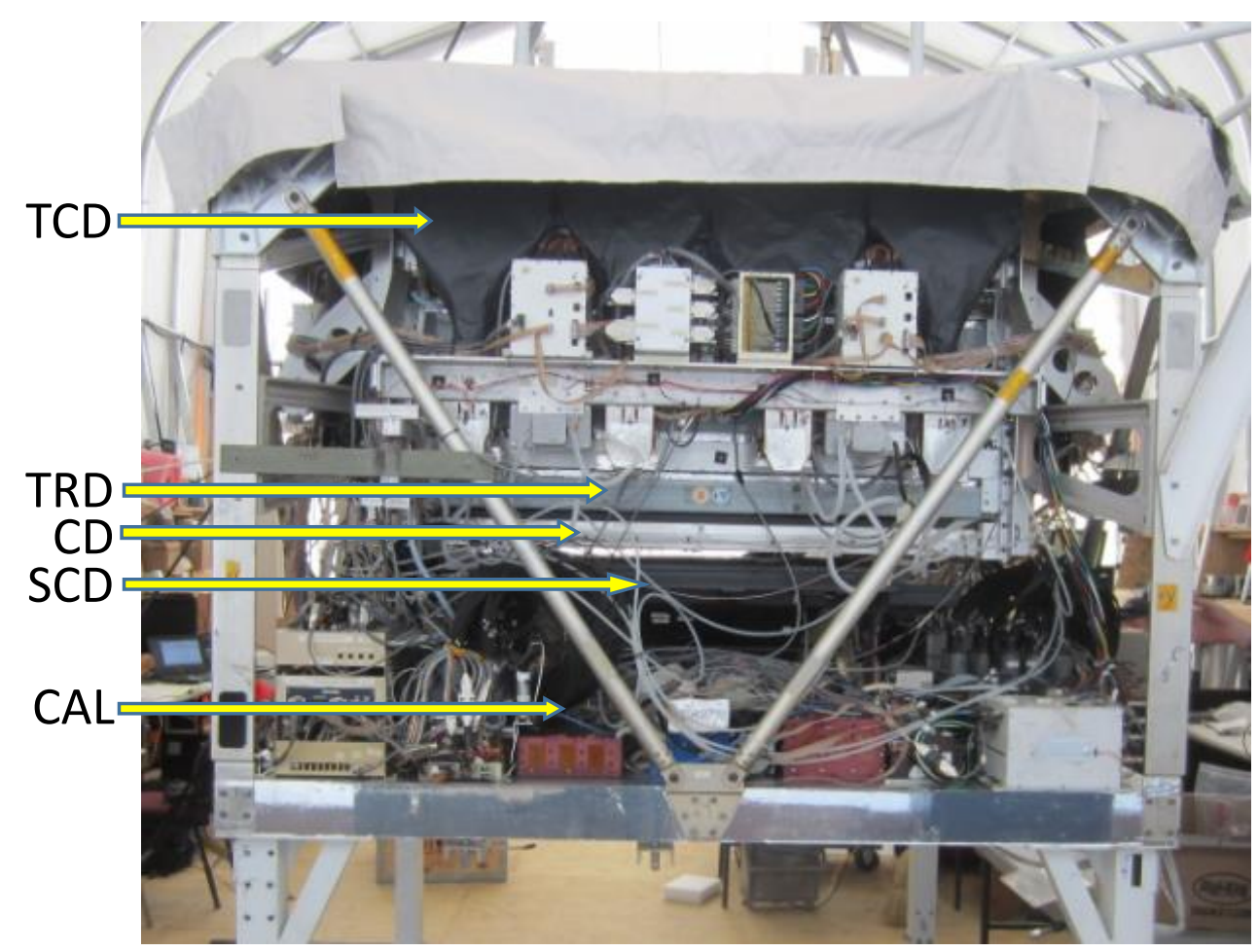

Fig. 1 BACCUS Instrument

\section{Instrument and performance}

\subsection{TCD, S3 and CD}

The TCD, for trigger and charge measurement, consist of two orthogonal layers of four 5 $\mathrm{mm}$ thick plastic scintillator paddles each covering $120 \mathrm{~cm} \times 120 \mathrm{~cm}$. The paddles are read out by fast photomultiplier tubes (PMTs) via twisted-strip adiabatic light guides. The TCD measures the light yield produced by the particle in plastic scintillator [3].

The S3 scintillating square fibers $\left(2 \times 2 \mathrm{~mm}^{2}\right)$ are read out by two PMTs and is located just above the calorimeter, and provides a reference time for TCD timing readout. 
The $\mathrm{CD}$ has a $1 \mathrm{~cm}$ thick plastic radiator with $1 \mathrm{~m}^{2}$ surface area and is instrumented with eight PMTs viewing wavelength shifting bars placed along the radiator edges. It is used to flag relativistic particles to provide a charge determination complementary to that of the TCD. The $\mathrm{CD}$ responds only to particles with velocity exceeding the velocity of light in the plastic. It allows BACCUS to reject the abundant low energy cosmic rays present in the polar region. Fig. 2 shows the ground muon peak around 750 ADC from one of the TCD's 16 PMTs in performance testing.

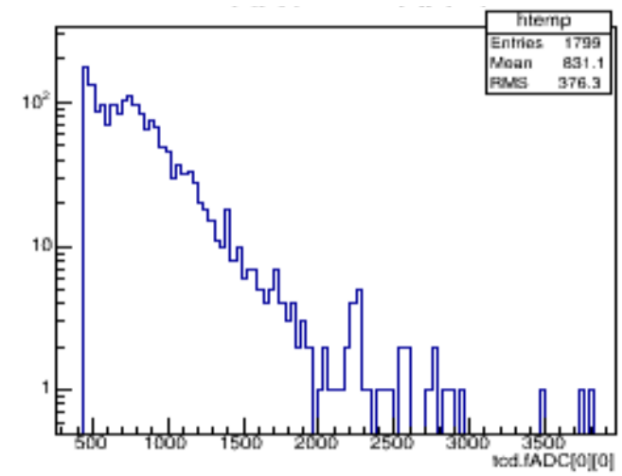

Fig. 2. Ground muon peak in the TCD in units of ADC.

\subsection{TRD}

The TRD, for energy measurement, is based on the gas straw technique and is new for the BACCUS flight, compared to the CREAM-I TRD. The $1200 \mathrm{~mm}$ long thin-walled gas proportional straw tubes, of $10 \mathrm{~mm}$ diameter, are filled with $\mathrm{Xe} / \mathrm{Co}_{2} 80 / 20 \%$ gas mixture at 1 atmosphere. The TRD consists of 8 modules (organized in two sections) with alternating orthogonal straw orientations. Each module contains 200 straw tubes arranged in dense double layers and a TR radiator made of $50 \mathrm{~mm}$ thick plastic foam material. The High Voltage (HV) modules are placed inside the light aluminium gas manifolds to avoid exposure to the low pressure environment at float altitude [4]. The TRD was calibrated with $\mathrm{e}^{-}(100 \mathrm{GeV})$ and $\pi^{+}$ $\left(250\right.$ and $350 \mathrm{GeV}$ ) beams at CERN. Fig. 3 shows the clear peak at around $8200 \mathrm{ADC}$ of the $\pi^{+}$ $350 \mathrm{GeV}$ beams in one TRD channel [5].

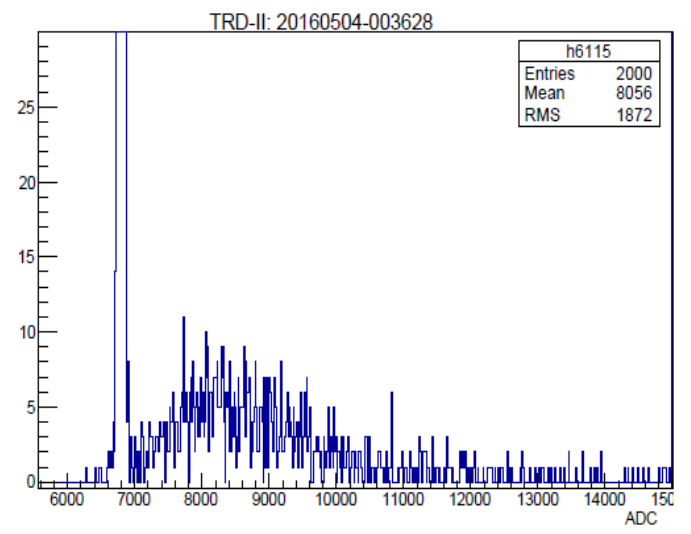

Fig. 3. The response of one TRD channel from the $\pi^{+} 350 \mathrm{GeV}$ beams.

\subsection{SCD}

The SCD, for charge measurement, is composed of two layers of 156 silicon sensors each with thickness $380 \mu \mathrm{m}$, segmented into an array of 4 x 4 pixels, each with an active area of 
$2.12 \mathrm{~cm}^{2}$ [6]. The sensors are slightly tilted and overlap each other in both lateral directions, providing a full coverage in a single layer and covering an area of $77.9 \times 79.5 \mathrm{~cm}^{2}$. The dual layers, about $4 \mathrm{~cm}$ apart, cover an effective inner area of $0.52 \mathrm{~m}^{2}$ with no insensitive regions. The readout electronics of the 4992 pixels is based on a 16-channel ASIC chip followed by 16bit analog-to-digital converters (ADCs), providing a fine charge resolution over a wide dynamic range from hydrogen to nickel. Fig. 4 shows charge gains of the top SCD with the DAC/ADC values of $~ 1.9$ in most channels which is consistent with the previous flight data.

Multiple charge measurements with TCD, CD and SCD accurately identify the incident particles by minimizing the effect of backscattered particles from the calorimeter. The TCD is based on the fact that the incident particles enter the TCD before developing a shower in the calorimeter, while the backscattered particles arrive several nanoseconds later. The segmented SCD pixels minimize multiple hits of backscattered particles in a segment.

\subsection{CAL}

The CAL, for trigger and energy measurement, is a stack of 20 tungsten plates, each 1 radiation length $\left(\mathrm{X}_{0}\right)$ thick and with a surface area $50 \times 50 \mathrm{~cm}^{2}$, interleaved with layers of $1 \mathrm{~cm}$ wide and $50 \mathrm{~cm}$ long scintillating-fiber ribbons. Each ribbon is built by gluing together 19 scintillating fibers of $0.5 \mathrm{~mm}$ diameter each. The light signal from each ribbon is collected by means of an acrylic light mixer coupled to a bundle of 48 clear fibers where each fiber is distributed to low, mid, and high range channels. A total of 2560 channels are read out from 40 Hybrid Photodiodes (HPDs) powered in groups of 5 units. The use of both TRD and CAL instruments allows in-flight cross-calibration of the two techniques and, consequently, provides a powerful method for measuring cosmic-ray energies. CAL was calibrated at CERN with $\mathrm{e}$ $(100 \mathrm{GeV})$ and $\pi^{+}(250$ and $350 \mathrm{GeV})$ beams at CERN in 2015 and 2016. Additionally, the pedestal, charge gain and HPD response to LED light were tested. The BACCUS calorimeter average RMS value of the pedestal was about 11 ADC as shown in Fig. 5 and was consistent with the previous flight values. Pedestal levels and charge gains were stable and all HV channels responded to the LED signals in the performance test [7].

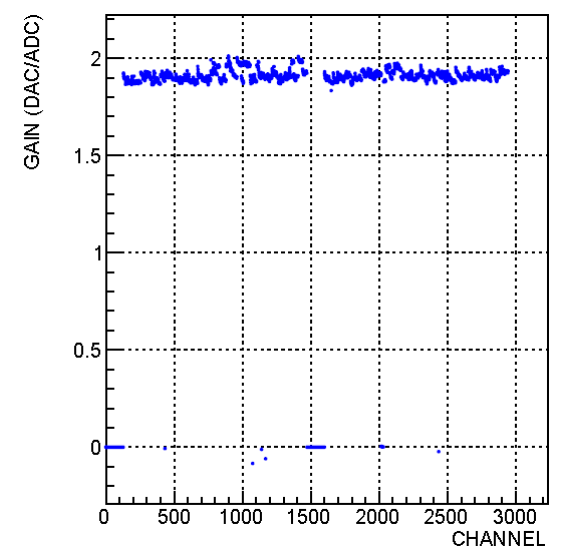

Fig. 4. Charge Gain runs with the Top SCD. Fig. 5. CAL pedestal response

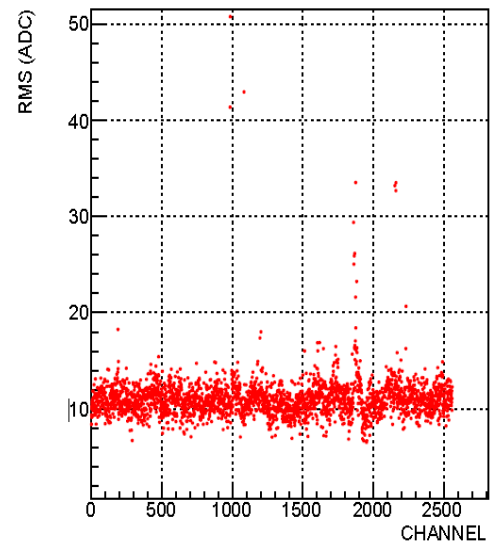

\section{Flight}

BACCUS was launched in Antarctica on Nov. 28, 2016 and landed on Dec. 28, 2016 after a 30 day flight. The balloon altitude was between 36 and $40 \mathrm{~km}$ throughout most of the flight. 
The corresponding average atmospheric overburden was only $\sim 3.9 \mathrm{~g} / \mathrm{cm}^{2}$. The temperature of the various instrument boxes stayed within the required operational range with daily variation of a few ${ }^{\circ} \mathrm{C}$, consistent with the Sun angle as shown in Fig. 6. To assure proper sparsification thresholds and pedestal subtraction despite this thermal drift, pedestal runs were performed once every hour for calorimeter and every 20 minutes for SCD and TRD throughout the flight, leading to a pedestal value drift of less than $1 \mathrm{ADC}$ count between two consecutive pedestal runs. All the high energy data $(>\sim 1 \mathrm{TeV})$ were transmitted via TDRSS during the flight, while the lower energy data were recorded on board. A total of about 70 GB data were collected from the flight.

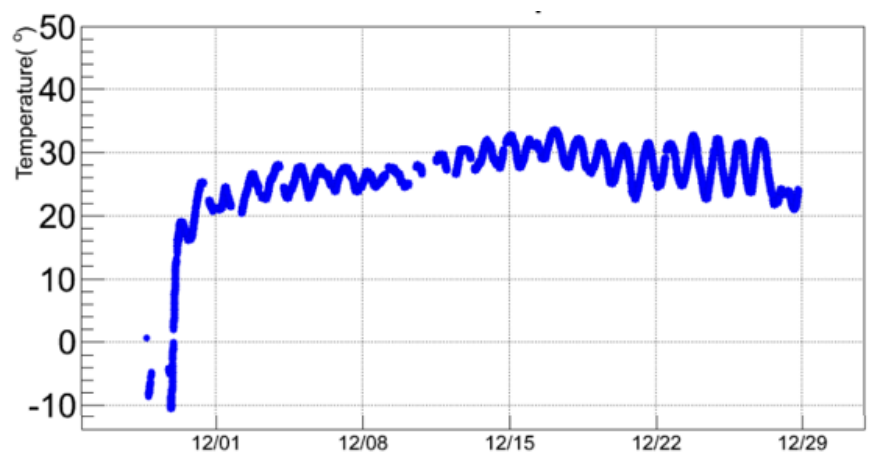

Fig. 6. The temperature variation in ${ }^{\circ} \mathrm{C}$ of a calorimeter HPD box during the flight

\section{Summary}

The balloon-borne BACCUS experiment directly measures the elemental spectra of cosmicray nuclei from protons to iron over the energy ranges $\sim 10^{12}$ to $10^{15} \mathrm{eV}$. It focuses on energy dependence of secondary to primary ratios (e.g. B/C) to investigate cosmic-ray propagation history as well as acceleration mechanisms and their sources.

BACCUS consists of redundant and complementary particle detectors including the TCD, TRD, CD, SCD, S3 and CAL. Each subdetector was tested and showed reasonable performance. BACCUS was calibrated at CERN in April, 2016. It was integrated with SIP at CSBF and all performance tests were completed. The 30 day flight was carried out over the Antarctica collecting data 70 GB in 2016 from Nov. 28 to Dec. 28 and the cumulative flight duration reached 191 days together with previous CREAM balloon flights. The payload was recovered from the flight, refurbished, and has been integrated in preparation for a next flight.

\section{Acknowledgement}

This work was supported in the US by NASA grants NNX11AC52G, NNX08AC15G, NNX08AC16G and their predecessor grants in Korea by the Creative Research Initiatives of MEST/NRF. We also greatly appreciate the support of the Columbia Scientific Balloon Facility (CSBF), the National Science Foundation (NSF) Office of Polar Program, and the Antarctic Support Contract (ASC) for their support of the flight campaigns in Antarctica.

\section{References}

[1] E. S. Seo et al., Cosmic-ray energetics and mass (CREAM) balloon project, Adv. Sp. Res. 33. 10, 17777-1785 (2004) 
[2] H. S. Ahn et al., Energy Spectra of Cosmic-ray Nuclei at High Energies, APJ, 707, 593 (2009)

[3] S. Coutu et al., Design and performance in the first flight of the transition radiation detector and charge detector of the CREAM balloon instrument, Nucl. Inst. Meth. A, 572, 485-487 (2007)

[4] A. Malinin et al., A New Transition Radiation Detector for the CREAM experiment, Proc. of $32^{\text {nd }}$ ICRC, Beijing, 6, 408 (2011)

[5] N. Picot-Clemente et al., Performance of the BACCUS Transition Radiation Detector, Proc. of $35^{\text {th }}$ ICRC, Busan (2017) in submitting

[6] I. H. Park, et al., Silicon Charge Detector for the CREAM experiment, Nucl. Instr. Meth. Phys. Res. A, 570, 286 (2007)

[7] J. H. Han et al., Performance of the CREAM-V and CREAM-VI calorimeters in flight, Proc. of $32^{\text {nd }}$ ICRC, Beijing, 6, 388 (2011) 